\title{
Geometric parameter study of piezoelectric coverage on a rectangular cantilever energy harvester
}

\author{
R Patel, S McWilliam and A A Popov \\ Materials, Mechanics and Structures Research Division, Faculty of Engineering, \\ University of Nottingham, Nottingham, NG7 2RD, UK \\ E-mail: eaxrp@nottingham.ac.uk
}

\begin{abstract}
This paper proposes a versatile model for optimising the performance of a rectangular cantilever beam piezoelectric energy harvester used to convert ambient vibrations into electrical energy. The developed model accounts for geometric changes to the natural frequencies, mode shapes and damping in the structure. This is achieved through the combination of Finite Element (FE) modelling and a distributed parameter electromechanical model, including load resistor and charging capacitor models. The model has the potential to investigate the influence of numerous geometric changes on harvester performance, and incorporates a model to account for changes in damping as the geometry changes. The model is used to investigate the effects of substrate and piezoelectric layer length, and piezoelectric layer thickness on the performance of a MEMS-scale device. Findings from a parameter study indicate the existence of an optimum sample length due to increased mechanical damping for longer beams and improved power output using thicker piezoelectric layers. In practice, harvester design is normally based around a fixed operating frequency for a particular application, and improved performance is often achieved by operating at or near resonance. To achieve unbiased comparisons between different harvester designs, parameter studies are performed by changing multiple parameters simultaneously with the natural frequency held fixed. Performance enhancements were observed using shorter piezoelectric layers compared to the conventional design, in which the piezoelectric layer and substrate are of equal length.
\end{abstract}

Energy harvesting, Piezoelectric beam, Finite element method 


\section{Introduction}

Vibration energy harvesting has received significant research interest in recent years. The concept has the potential to replace batteries in applications such as wireless sensors, e.g. a tyre pressure monitoring sensor [1]. The three main transduction mechanisms are: piezoelectric, electromagnetic [2] and electrostatic [3], and of these piezoelectric devices have received most interest. Manufacture can be integrated readily into existing Micro-Electro-Mechanical Systems (MEMS) fabrication techniques [4] and the maximum energy storage density exceeds the other transduction mechanisms [5]. A common issue with electromagnetic devices is their low output voltage, requiring the use of a step-up transformer to assist integration with the power electronics. A drawback with electrostatic devices is their inability to function without a separate priming voltage. Piezoelectric devices are not limited by such problems, increasing their appeal to researchers. Further information regarding vibration energy harvesting can found in [6].

The conventional design for a MEMS-scale piezoelectric energy harvester is based on a cantilever beam configuration, consisting of a substrate layer with attached piezoelectric layers (e.g. lead zicronate titanate (PZT)) and a tip mass to tune the natural frequency of the beam. Some research has been performed to determine the optimum design configuration for piezoelectric cantilever energy harvesters. Lin et al [7] investigated how geometric parameters such as beam length and substrate dimensions affect the theoretical performance of a MEMS-scale rectangular cantilever beam, and found that the generated power could be improved by using shorter beams with thicker substrate layers. Gao et al [8] carried out a parameter study to determine the optimum length of the piezoelectric layer, and their model indicates that a device with a piezoelectric layer that is shorter than the substrate layer would outperform the conventional design, in which the piezoelectric layer has the same length as the substrate. In both above studies the influence of the piezoelectrical layer on the mechanical properties of the system was neglected. This could lead to the beam displacement and power generated being over-estimated. Also, the mechanical excitation used in Gao et al [8] was provided by applying a point force at the cantilever tip - a scenario that is unlikely to occur in practice. In addition, the power circuit was modelled as a load resistance, and no mention was made how the magnitude of the load resistance affects the power output. The authors of this paper have found that the load resistance is an important factor in the relationship between geometric parameters and power output. Other relevant work includes Friswell and Adhikari [9] in which the optimum shape of the piezoelectric material electrode is determined by varying the width of the material along the length of the beam. Their results indicate a $115 \%$ increase in power output is achievable if the electrode is designed to cover roughly half the piezoelectric material. In their work the load resistance is kept constant at $100 \mathrm{k} \Omega$; if a range of magnitudes had been examined then different trends would occur. Chen et al [10], theoretically and experimentally, examine three different shapes of cantilever - rectangular, trapezoidal 
and triangular. Experimental findings show a gain in excess of 250\%, for peak voltage, is achievable when utilising a triangular cantilever due to improved stain distribution along the beam length. Other recent works on geometric parameter studies include Zhu et al [11] and Bourisli and Al-Ajmi [12]. The trends presented in these papers will be compared to the trends presented later in this study.

All previous work on geometric parameter studies has focused on determining trends when only a resistive load is used in the electrical circuit. In the majority of practical applications the direct power generated by a harvester is insufficient to power the device, and it is necessary to store the generated energy in some form [13]. In this work, a more realistic electrical circuit, consisting of a diode bridge for rectification and a capacitor for energy storage is also considered in the modelling, together with a rigorous model of the piezoelectric coupling.

The purpose of this paper is to gain understanding of the influence of geometric parameters on the performance of a piezoelectric cantilever MEMS-scale energy harvester undergoing harmonic base excitation. This is achieved through the development and validation of an analytical model for a cantilever beam piezoelectric energy harvester, and a detailed parameter study. Section 2 presents the analytical and finite element modelling techniques used to determine and compare the performance of energy harvesters with different geometries. It is necessary to incorporate geometric changes in a consistent way within the modelling. After reviewing previous parameter studies, the authors identified the need to consider the effect of geometric changes on natural frequencies, mode shapes and damping. Section 3 presents various parameter studies that investigate the influence of individual geometric parameters on the power output across a load resistor. The effect of geometric parameters on the natural frequency is particularly important, as the performance of the harvester is maximised when the base excitation frequency is close to the natural frequency. To take account of this fact and achieve a fair comparison of results, some of the parameter studies performed later consider geometric parameter variations with the natural frequency held fixed. In each case, the trends for the power output across the load resistor are discussed. Lastly the effects of geometric parameters on the voltage stored across a capacitor are examined. The paper closes with a summary of its findings.

\section{Modelling the energy harvester system}

\subsection{Energy harvester}

Various models have been developed to predict the performance of a piezoelectric cantilever beam energy harvester [14, 15]. Erturk and Inman [16] have shown that earlier single-degree-of-freedom models, e.g. Williams and Yates [14], were inaccurate

because of limitations in the modelling of the cantilever beam dynamics, the piezoelectric coupling in the mechanical domain, and the base excitation. The distributed-parameter electromechanical model developed by Erturk and Inman [17] provides an accurate 


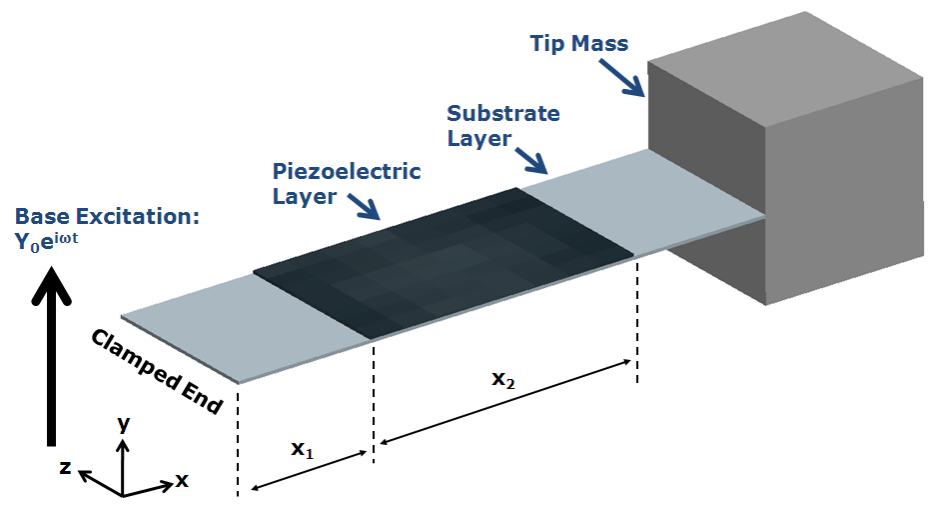

(a) Isometric view

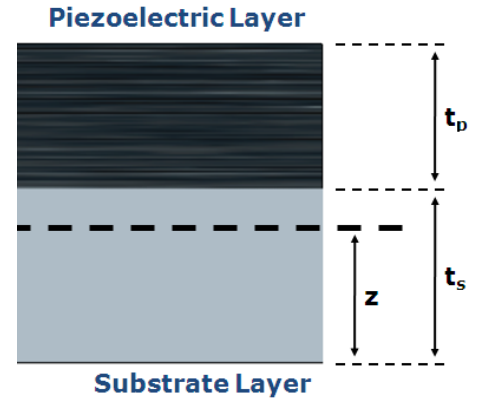

(b) Side view of two layers

Figure 1: Schematic representation of an energy harvester.

mathematical model of the energy harvester which has been validated experimentally [18]. This model will be used as the basis for the energy harvester model developed in this paper.

The piezoelectric harvester considered consists of a composite two-layer EulerBernoulli beam, with piezoelectric material perfectly bonded to a substrate layer, as shown in Figure 1. The mechanical behaviour of the system can be analysed using modal analysis techniques, such that the beam deflection $w_{\text {rel }}(x, t)$ is expressed as:

$$
w_{\text {rel }}(x, t)=\sum_{r=1}^{\infty} W_{r}(x) \eta_{r}(t),
$$

where $W_{r}(x)$ is the mass-normalised eigenfunction of the $r^{\text {th }}$ mode of the structure, and $\eta_{r}(t)$ is the associated modal coordinate. Using Erturk and Inman [17], the equation governing the modal coordinates is given by:

$$
\ddot{\eta}_{r}(t)+2 \gamma_{r} \omega_{r} \dot{\eta}_{r}(t)+\omega_{r}^{2} \eta_{r}+R_{r} v(t)=Q_{r}(t),
$$

where $v(t)$ is the voltage generated by the piezoelectric material, and $\gamma_{r}, \omega_{r}, R_{r}$ and $Q_{r}$ are damping ratio, natural frequency, backward coupling term and forcing term for the $r^{\text {th }}$ mode, respectively. In the derivation of Equation (2), the length and position of the piezoelectric layer relative to the substrate is taken into account using the Heaviside function $\left[H\left(x-x_{1}\right)-H\left(x-x_{2}-x_{1}\right)\right]$, where lengths $x_{1}$ and $x_{2}$ are defined in Figure 1(a). In addition, the mass-normalised eigenfunction and natural frequency must also account for the length and position of the piezoelectric layer and this is achieved using the finite element method described in Section 2.2.

For harmonic base acceleration $Y_{0} \omega^{2} \mathrm{e}^{\mathrm{i} \omega t}$, the equations defining $Q_{r}$ and $R_{r}$ are given by:

$$
\begin{gathered}
Q_{r}(t)=Y_{0} \omega^{2} \mathrm{e}^{\mathrm{i} \omega t} \int_{0}^{L} m(x) W_{r}(x) \mathrm{d} x \\
R_{r}(t)=-\frac{E_{p} b_{p} d_{31}}{2 t_{p}}\left[\left(t_{p}+t_{s}-z\right)^{2}-\left(t_{s}-z\right)^{2}\right]\left[W_{r}^{\prime}\left(x_{2}+x_{1}\right)-W_{r}^{\prime}\left(x_{1}\right)\right],
\end{gathered}
$$


where $Y_{0}$ is the base displacement magnitude in the y direction, $\omega$ is the frequency of excitation, $L$ is the total beam length, and $m(x)$ is the mass per unit length. $E_{p}, b_{p}$, $t_{p}$ denote the Young's modulus, width and thickness, respectively, for the piezoelectric material. $t_{s}$ is the thickness of the substrate layer, and $z$ is the distance between the neutral axis of the composite area and the bottom of the substrate layer, see Figure 1(b).

As the geometry of the energy harvester is altered the mechanical damping ratio $\gamma_{r}$ of the system will change. To incorporate this change, the damping is assumed to be of proportional type, such that:

$$
\gamma_{r}=\frac{\alpha}{2 \omega_{r}}+\frac{\beta \omega_{r}}{2}
$$

where $\alpha$ and $\beta$ are constants obtained from experimental frequency response data.

The next step is to obtain an expression for the current generated by the piezoelectric material and this is achieved by considering the following piezoelectric constitutive equation:

$$
D_{3}=e_{31} S_{1}+\epsilon_{33}^{S}\left(E_{\text {field }}\right)_{3},
$$

where $D_{3}$ is the electric displacement, $e_{31}$ is a piezoelectric constant of units $\mathrm{C} / \mathrm{m}^{2}, S_{1}$ is the strain, $\epsilon_{33}^{S}$ is the permittivity at constant stress of units $\mathrm{F} / \mathrm{m}$, and $\left(E_{\text {field }}\right)_{3}$ is the electric field strength. Index 3 refers to the y-direction, i.e. through the thickness of the material, and index 1 refers to the x-direction, i.e. parallel to the beam length. Assuming the electric field is uniform throughout the piezoelectric material thickness, $t_{p}$, then $\left(E_{\text {field }}\right)_{3}(t)$ can be expressed as $-v(t) / t_{p}$, where $v(t)$ is the voltage across the piezoelectric material, see also Equation (2). In addition, expressing the piezoelectric constant $e_{31}$ as $d_{31} E_{p}$ yields the relation:

$$
D_{3}(x, t)=d_{31} E_{p} S_{1}(x, t)-\epsilon_{33}^{S} \frac{v(t)}{t_{p}} .
$$

Strain in the piezoelectric material can be obtained by considering the curvature of the beam and the distance between the centre of the piezoelectric material and the composite neutral axis, $t_{p n},[19]$ :

$$
S_{1}(x, t)=-t_{p n} w_{\text {rel }}^{\prime \prime}(x, t) .
$$

The electric displacement is then integrated over the electrode area to determine the electric charge generated by the piezoelectric material [17]:

$$
q(t)=\int_{A} \mathbf{D} \cdot \mathbf{n d} \mathbf{A},
$$

where $\mathbf{D}$ is a vector of electric displacements and $\mathbf{n}$ is the unit outward normal. A slight alteration is made to the method in [17] whereby the electrode area is realised here using $x_{1}$ and $x_{2}$. An expression for the generated current, defined as the rate of flow of charge - $\mathrm{d} q(t) / \mathrm{d} t$ is obtained by combining Equations (7), (8) and (9):

$$
i(t)=-\int_{x_{1}}^{x_{2}+x_{1}} d_{31} E_{p} t_{p n} b_{p} \dot{w}_{\text {rel }}^{\prime \prime}(x, t)-C_{p} \dot{v},
$$


where $C_{p}$ represents the piezoelectric internal capacitance given by

$$
C_{p}=\frac{\epsilon_{33}^{S} b_{p} x_{2}}{t_{p}} .
$$

Initial results will be obtained for power dissipated, $P$, across a resistor having resistance $R_{\text {load }}$, and this can be obtained using $P=v(t)^{2} / R_{\text {load }}$, where $v(t)=i(t) R$. If we now use the method of separation of variables and define the forward coupling term, $\phi$, as

$$
\phi_{r}=\frac{-d_{31} E_{p}\left(0.5 t_{p}+t_{s}-z\right) b_{p}}{C_{p}}\left[W_{r}^{\prime}\left(x_{2}\right)-W_{r}^{\prime}\left(x_{1}\right)\right],
$$

the resulting differential equation for the generated voltage is:

$$
\dot{v}(t)+\frac{1}{C_{p} R_{\text {load }}} v(t)=\sum_{r=1}^{\infty} \phi_{r} \dot{\eta}_{r}(t) .
$$

For harmonic base excitation, the voltage generated is assumed to be harmonic, $v(t)=V_{o} \mathrm{e}^{\mathrm{i} \omega t}$, along with the motion of the beam, and so the steady-state solution for Equation (2) can be expressed as:

$$
\eta_{r}(t)=\frac{Q_{r}-R_{r} V_{o}}{\left(\omega_{r}^{2}-\omega^{2}\right)+2 \gamma_{r} \omega_{r} \omega \mathrm{i}} \mathrm{e}^{\mathrm{i} \omega t} .
$$

Equations (1), (13) and (14) can be used to obtain expressions for the voltage generated by the energy harvester and the relative displacement of the beam. For the scenario when the harvester is excited close the fundamental frequency, $\omega_{1}$, only contributions from the first structural mode are required and the mechanical and electrical responses are given by:

$$
\begin{aligned}
& v(t)=\left[\frac{\frac{Q_{1} \phi_{1} \mathrm{i} \omega}{\left(\omega_{1}^{2}-\omega^{2}\right)+\left(2 \gamma_{1} \omega_{1} \omega\right) \mathrm{i}}}{\frac{R_{1} \phi_{1} \mathrm{i} \omega}{\left(\omega_{1}^{2}-\omega^{2}\right)+\left(2 \gamma_{1} \omega_{1} \omega\right) \mathrm{i}}+\left(\mathrm{i} \omega+\frac{1}{C_{p} R_{\text {load }}}\right)}\right] \mathrm{e}^{\mathrm{i} \omega t}, \\
& w_{\text {rel }}(x, t)=W_{1}(x)\left[\frac{Q_{1}-R_{1} V_{o}}{\left(\omega_{1}^{2}-\omega^{2}\right)+\left(2 \gamma_{1} \omega_{1} \omega\right) \mathrm{i}}\right] \mathrm{e}^{\mathrm{i} \omega t} .
\end{aligned}
$$

The results considered in the parameter study later are based on calculations performed using Equations (15) and (16), with accurate estimation of modal parameters from finite element simulations.

\subsection{Finite element model}

The model presented in Section 2.1 requires the natural frequencies and mode shapes of the piezoelectric cantilever beam shown in Figure 1. In this work a finite element

model is used to model the cantilever, taking into account the length and position of the piezoelectric layer. In this approach, the beam is split into three sections which in 


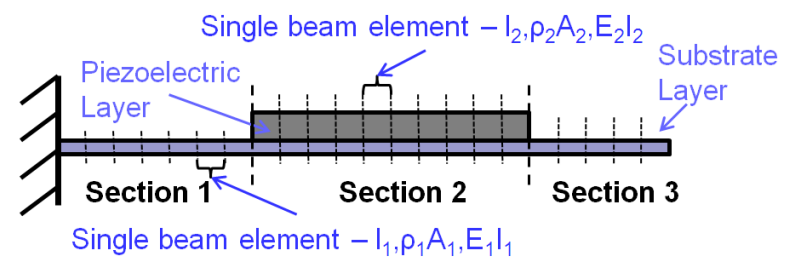

Figure 2: Element properties for beam sections.

turn are divided into elements. Substrate material solely makes up sections 1 and 3 while section 2 comprises of both piezoelectric and substrate material. The elements are assumed to be beam elements defined according to Euler-Bernoulli beam theory [19] with the element properties for the middle section differing from the other two sections, see Figure 2.

Each element has 4 degrees of freedom (transverse translation and rotation at each end) and the mass and stiffness matrices [19] for the $i^{\text {th }}$ element are given by:

$$
\begin{aligned}
& {\left[m_{i}\right]=\frac{\rho_{i} A_{i} l_{i}}{420}\left[\begin{array}{cccc}
156 & 22 l_{i} & 54 & -13 l_{i} \\
22 l_{i} & 4 l_{i}{ }^{2} & 13 l_{i} & -3 l_{i}{ }^{2} \\
54 & 13 l_{i} & 156 & -22 l_{i} \\
-13 l_{i} & -3 l_{i}{ }^{2} & -22 l_{i} & 4 l_{i}{ }^{2}
\end{array}\right],} \\
& {\left[k_{i}\right]=\frac{2 E_{i} I_{i}}{l_{i}^{3}}\left[\begin{array}{cccc}
6 & 3 l_{i} & -6 & 3 l_{i} \\
3 l_{i} & 2 l_{i}{ }^{2} & -3 l_{i} & l_{i}{ }^{2} \\
-6 & -3 l_{i} & 6 & -3 l_{i} \\
3 l_{i} & l_{i}{ }^{2} & -3 l_{i} & 2 l_{i}{ }^{2}
\end{array}\right]}
\end{aligned}
$$

where $\left[m_{i}\right]$ is the element mass matrix, $\left[k_{i}\right]$ is the element stiffness matrix, $E_{i}$ is the element Young's modulus, $I_{i}$ is the element area moment of inertia, $\rho_{i}$ is the element mass density, $A_{i}$ is the element cross-sectional area and $l_{i}$ is the length of the element.

The middle section is a composite piezoelectric/substrate beam and the stiffness of the elements in this section can be calculated using the composite neutral axis, defined by $z$ (see Figure 1(b)), where:

$$
z=\frac{\sum A y}{\sum A}=\frac{\left(t_{p} n b_{p}\right)\left(t_{s}+0.5 t_{p}\right)+\left(t_{s} b_{s}\right)\left(0.5 t_{s}\right)}{t_{p} n b_{p}+t_{s} b_{s}} .
$$

In this equation $t$ is the layer thickness, $b$ is the layer width and $n$ is $E_{p} / E_{s}$ (i.e. the Young's modulus for the piezoelectric layer divided by the Young's modulus of the substrate layer).

The equivalent flexural rigidity of the composite beam is given by:

$$
(E I)_{\text {composite }}=E_{s} I_{z s}+E_{p} I_{z p},
$$

where $I_{z s}$ and $I_{z p}$ are given by:

$$
I_{z s}=\frac{b_{s} t_{s}{ }^{3}}{12}+b_{s} t_{s}\left(z-\frac{t_{s}}{2}\right)^{2},
$$




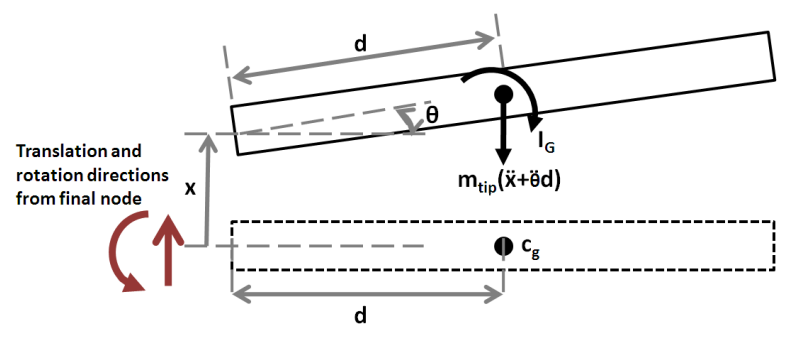

Figure 3: Effect of adding a tip mass on the final node's rotation and translation.

$$
I_{z p}=\frac{b_{p} t_{p}^{3}}{12}+b_{p} t_{p}\left(\frac{t_{p}}{2}+t_{s}-z\right)^{2} .
$$

The mass and stiffness matrices for individual elements can be assembled to generate global mass and stiffness matrices for the complete structure. The assembly process takes account of the boundary conditions at the clamped end, by setting the displacement and rotation of the clamped node to zero. The presence of the tip mass, assumed to be a rigid body, at the free end also needs to be taken into account, and this is achieved by developing a mass matrix for the tip mass. The kinematics and resulting inertia forces for an offset tip mass are shown in Figure 3, as the beam end exhibits translational and rotational motion. By individually considering the effect of beam end translation and rotation, it can be shown that the mass matrix for the offset tip mass is given by:

$$
\left[m_{\text {tip mass }}\right]=\left[\begin{array}{cc}
m_{\text {tip }} & m_{\text {tip }} d \\
m_{\text {tip }} d & I_{\mathrm{G}}+m_{\text {tip }} d^{2}
\end{array}\right],
$$

where $m_{\text {tip }}$ is the mass of the tip mass, $I_{\mathrm{G}}$ is the moment of inertia of the tip mass about its centre of mass, and $d$ is the offset distance.

Once the global mass matrix $[M]$ and stiffness matrix $[K]$ have been generated, the natural frequencies and mode shape for the complete structure can be calculated by solving the following eigenvalue equation:

$$
\left[[K]-\omega^{2}[M]\right] U=0
$$

where $\omega$ are the natural frequencies and $U$ is a vector of displacements and rotations defining the mode shapes, such that:

$$
[U]=\left[u_{2}, \theta_{2}, u_{3}, \theta_{3}, u_{4}, \theta_{4}, \ldots \ldots, u_{\text {ele }+1}, \theta_{\text {ele }+1}\right]^{\mathrm{T}}
$$

where 'ele' is the total number of elements used to model the structure.

\subsection{Charging Circuit}

In conjunction with an electrical circuit consisting solely of a resistor, a model which allows for some form of energy storage is developed. The electrical circuit consists of the energy harvester, represented as a current source in parallel with a capacitor $\left(\mathrm{C}_{\mathrm{p}}\right)$, 


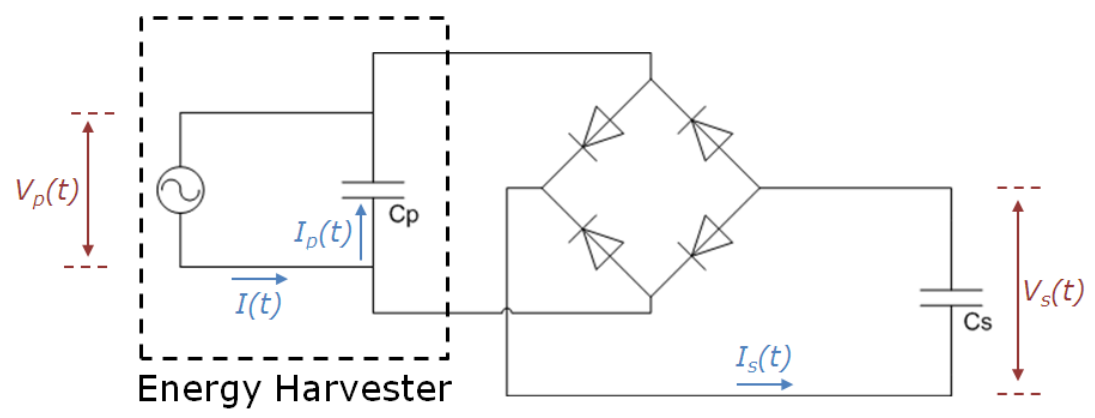

Figure 4: Schematic of the charging circuit.

a diode bridge for rectification and a second capacitor $\left(\mathrm{C}_{\mathrm{s}}\right)$ for storage, see Figure 4 . The following equations are used to model the electrical behaviour of the circuit:

$$
\begin{aligned}
& I_{p}(t)= \begin{cases}I(t) & \text { for } V_{p}(t)<V_{s}(t)+2 V_{t h} \\
I(t) \frac{C_{p}}{C_{s}+C_{p}} & \text { for } V_{p}(t) \geq V_{s}(t)+2 V_{t h}\end{cases} \\
& I_{s}(t)= \begin{cases}0 & \text { for } V_{p}(t)<V_{s}(t)+2 V_{t h} \\
I(t) \frac{C_{s}}{C_{s}+C_{p}} & \text { for } V_{p}(t) \geq V_{s}(t)+2 V_{t h}\end{cases}
\end{aligned}
$$

where $V_{t h}$ is the diode threshold voltage, assumed to be $0.6 \mathrm{~V}$, and $C_{s}$ is the storage capacitance, taken to be $1 \mathrm{nF}$. $I$ is the current generated by the piezoelectric material, $I_{p}$ and $I_{s}$ represent the currents flowing through $C_{p}$ and $C_{s}$, respectively. $V_{p}$ is the voltage across the piezoelectric material while $V_{s}$ is the voltage across the storage capacitor.

The electrical circuit is modelled in Simulink using components from the Simscape [20] and SimElectronics [21] libraries, see Figure 5 for the electrical subsystem. The Simulink model also incorporates the mechanical aspects of the system (not shown), allowing for electric domain coupling to remain.

\section{Model Validation and Numerical Results}

The dimensions and material properties for the structure considered, on which a parameter study is later performed, are shown in Table 1. This particular device was manufactured in 2006 by Fang et al [22] using MEMS fabrication techniques; Figure 6 shows a schematic representation of the device. The substrate layer is silicon, the piezoelectric material is PZT and the tip mass is nickel. Further details can be found in Fang et al [22]. The actual tip mass was bonded onto the top of the PZT layer. However, during the following analysis it is assumed that the tip mass overhangs the end of the beam, with its centre of mass coinciding with the neutral axis of the beam. This slight modification is likely to have some influence on the natural frequency and strain acting in the structure compared to the original, results in [22]. 


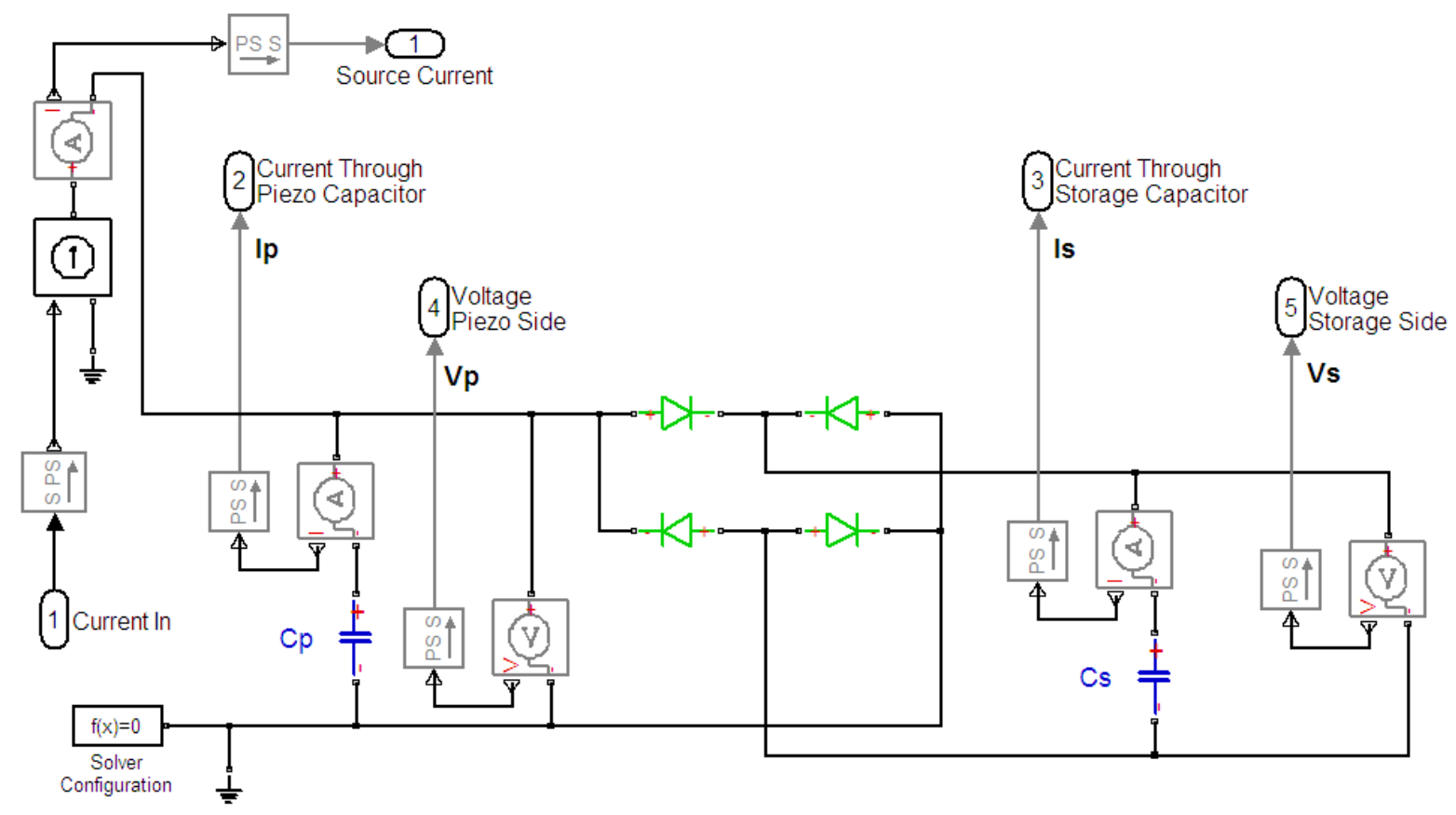

Figure 5: Model created in Simulink to represent an electrical system consisting of a storage capacitor in parallel with a resistor (assumed to have a magnitude of zero during this parameter study).

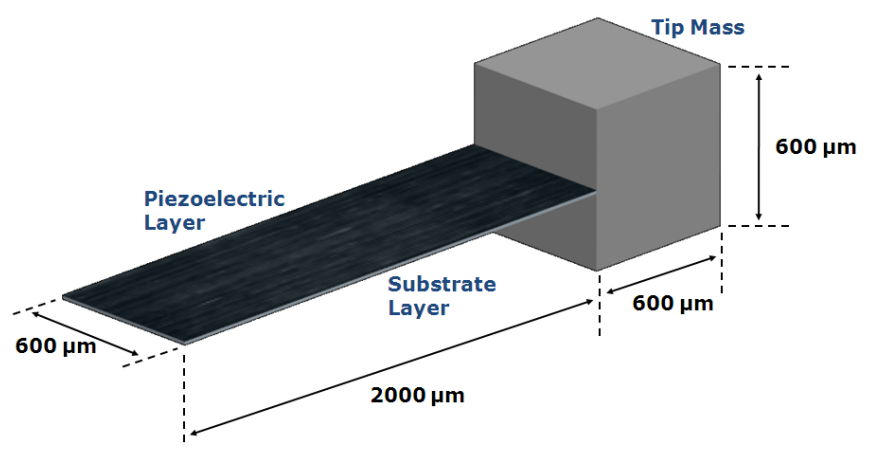

(a) Isometric view

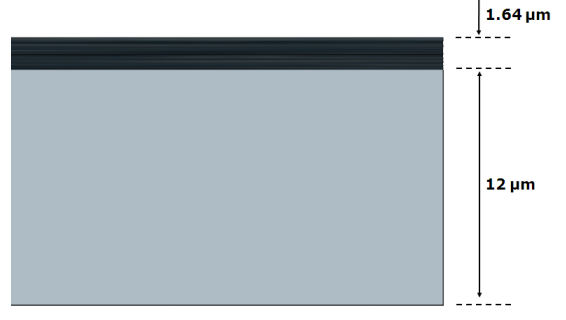

(b) Side view of the two layers

Figure 6: Schematic representation of the conventional harvester with dimensions.

Figure 7(a) shows a schematic of an ABAQUS [23] model used to validate the finite element approach presented in Section 2.2, using the nominal data presented in Table 1. The ABAQUS model was developed using 20-noded brick elements, and the results shown in Figure 7(b) indicate that the results obtained using the proposed finite element method and the ABAQUS method agree to within $1.6 \%$ for the predicted natural frequencies.

The overall energy harvester model has been validated previously against experimental measurements [24]. Samples were manufactured in-house using aluminium as the substrate layer and PZT as the piezoelectric layer. Adhesion and electrical contact on the underside of the piezoelectric layer were realised through a combination of DP460 


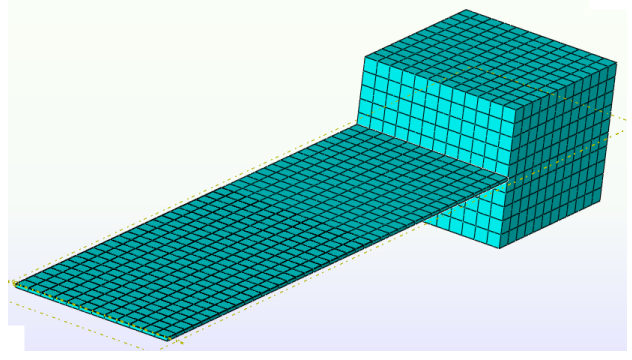

(a)

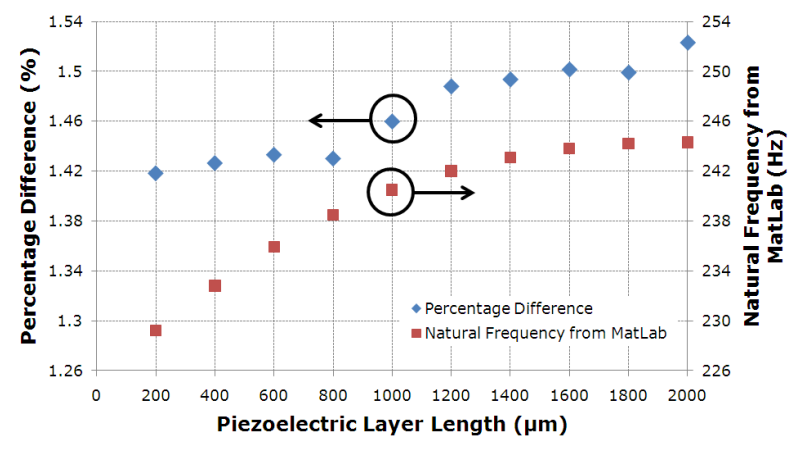

(b)

Figure 7: (a) - Schematic of Abaqus model. (b) - Plot showing percentage differences between the MatLab finite element model and Abaqus CAE for the natural frequency of harvester configurations with differing lengths of piezoelectric material. Also shown are the frequency magnitudes obtained from MatLab.

Table 1: Structural dimensions and mechanical properties of the conventional harvester used throughout this analysis.

\begin{tabular}{ll}
\hline Parameter & Magnitude \\
\hline Substrate length $(\mu \mathrm{m})$ & 2000 \\
Substrate width $(\mu \mathrm{m})$ & 600 \\
Substrate thickness $(\mu \mathrm{m})$ & $12^{\mathrm{a}}$ \\
PZT length $(\mu \mathrm{m})$ & $2000^{\mathrm{a}}$ \\
PZT width $(\mu \mathrm{m})$ & 600 \\
PZT thickness $(\mu \mathrm{m})$ & $1.64^{\mathrm{a}}$ \\
Tip mass length $(\mu \mathrm{m})$ & $600^{\mathrm{a}}$ \\
Tip mass width $(\mu \mathrm{m})$ & 600 \\
Tip mass thickness $(\mu \mathrm{m})$ & $600^{\mathrm{a}}$ \\
Young's modulus of substrate $(\mathrm{GPa})$ & 185 \\
Density of substrate $\left(\mathrm{kgm}^{-3}\right)$ & 2329 \\
Young's modulus of PZT $(\mathrm{GPa})$ & 66 \\
Density of PZT $\left.(\mathrm{kgm})^{-3}\right)$ & 7800 \\
Density of tip mass $\left(\mathrm{kgm}{ }^{-3}\right)$ & 8908 \\
Piezoelectric constant, $d_{31}(\mathrm{~m} / \mathrm{V})$ & $-180 \times 10^{-12}$ \\
Permittivity, $\epsilon^{\mathrm{S}}{ }_{33}(\mathrm{~F} / \mathrm{m})$ & $1.59 \times 10^{-8}$
\end{tabular}

${ }^{a}$ Parameter will be altered during proceeding analysis.

epoxy and silver conductive epoxy. Further information relating to the manufacturing and testing procedures used can be found in [24]. Figure 8, from [24], shows typical agreement between theoretical predictions and experimental measurements for the displacement frequency response function, with percentage differences less than $2.5 \%$.

For the purposes of conducting geometric parameter studies, the base acceleration is kept constant at $2 \mathrm{~g}$ and 50 elements are used in the finite element model to represent the whole beam. The piezoelectric layer is positioned with one end at the clamped 


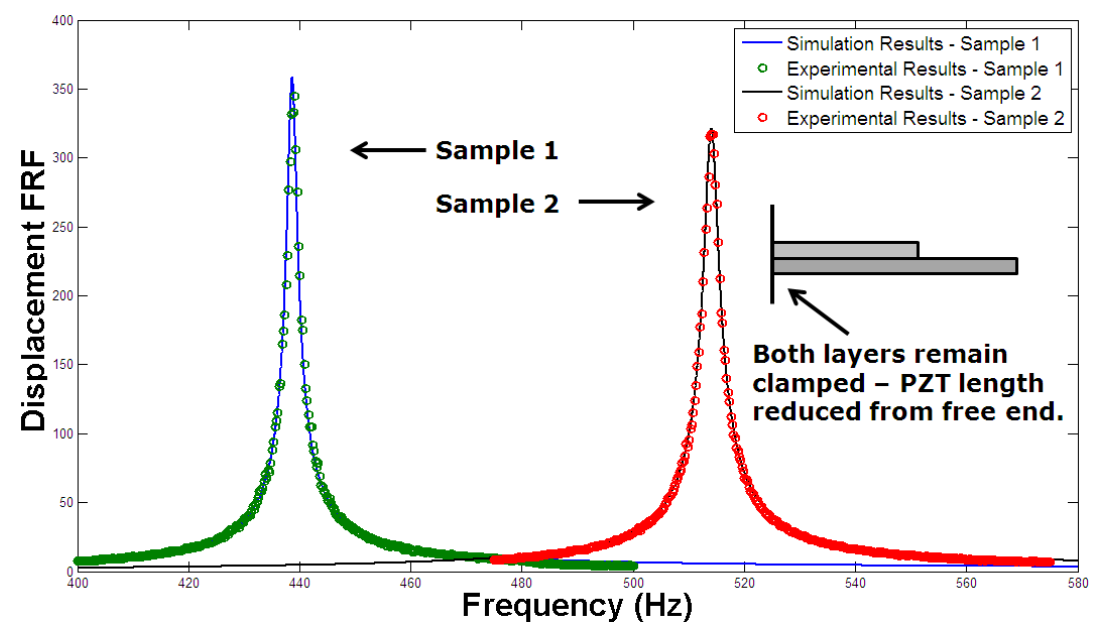

Figure 8: Comparison of experimental and theoretical results. Sample 1 consists of a $31.95 \mathrm{~mm} \times 5 \mathrm{~mm} \times 0.67 \mathrm{~mm}$ substrate layer and a $12 \mathrm{~mm} \times 5 \mathrm{~mm} \times 0.5 \mathrm{~mm}$ piezoelectric layer. Sample 2 was fabricated from a $24 \mathrm{~mm} \times 5 \mathrm{~mm} \times 0.67 \mathrm{~mm}$ substrate layer and a $21.49 \mathrm{~mm} \times 5 \mathrm{~mm} \times 0.5 \mathrm{~mm}$ piezoelectric layer, see [24] for details.

end (i.e. $\mathrm{x}_{1}=0$ ), so any reduction in piezo length is from the free end. In Section 3.1, the frequency of excitation, $\omega$, is chosen to be equal to the natural frequency, $\omega_{1}$, of the device. It is worth noting however that this approach does not guarantee that maximum power is achieved (see Figure 9). In addition, given that geometric changes will modify the natural frequency, a particular disadvantage of this approach is that the results do not correspond to the same excitation frequency. This can make interpretation of the results a little difficult. In Section 3.2 the constant-natural-frequency model is introduced. This is used in Section 3.3 to provide a consistent approach to comparing the performance of different harvesters. Multiple parameters are changed in order to keep the natural frequency fixed. In Section 3.1 effects of the following parameters are investigated: length of the energy harvester, the piezoelectric layer length and the piezoelectric layer thickness. Section 3.3 also looks at the effects of piezoelectric layer length and thickness but here the substrate thickness or tip mass inertia are used to control the natural frequency. In Section 3.4 results are presented using the capacitor charging circuit rather than the load resistor model used in Sections 3.1 to 3.3.

\subsection{Changing individual parameters}

The influence of individual geometric parameters on the power generated are considered in this sub-section. The model was first used to investigate the effect of changing the length of the cantilever, and Figure 10 shows the influence of length and load resistance on the power generated. The results indicate that as the length of a short beam is increased, higher power outputs are realised. However, as the length is increased further, the power output decreases. This behaviour can be explained by considering the influence of beam length on damping, see Figure 11. In the model developed, 


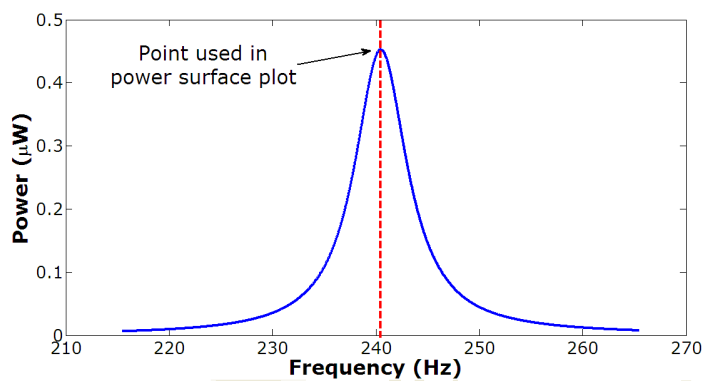

(a) Magnitude of load resistor $-5 \mathrm{k} \Omega$

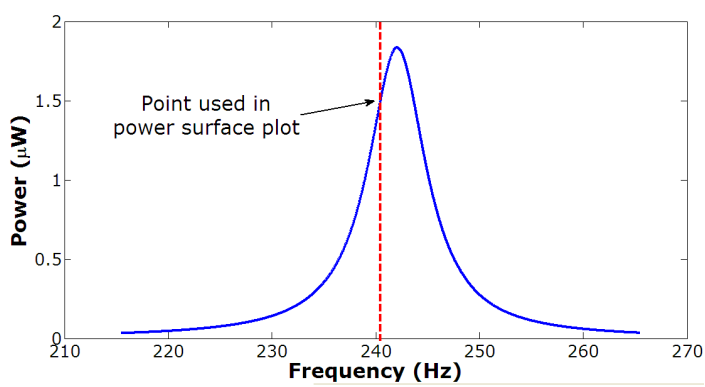

(b) Magnitude of load resistor - $500 \mathrm{k} \Omega$

Figure 9: Plots showing how the frequency at which maximum power occurs does not always coincide with the natural frequency of the device, red dotted line, 240.5 Hz. The length of the piezolayer in this configuration is $1000 \mu \mathrm{m}$, all other dimensions can be found in Table 1.

the damping ratio is calculated using Equation (5), where the constants $\alpha$ and $\beta$ were calculated using experimentally measured data. This was based on a macro-scale sample with equal length substrate and piezoelectric layers. The length and width of the layers were $49.5 \mathrm{~mm}$ and $5 \mathrm{~mm}$ respectively, whilst the thickness of the substrate and piezoelectric layer were $0.67 \mathrm{~mm}$ and $0.5 \mathrm{~mm}$, respectively. From the frequency response plot, the natural frequencies of the first two modes were determined to be $287.04 \mathrm{~Hz}$ and $1798.80 \mathrm{~Hz}$. Using the half-power method [19], the damping ratios corresponding to these modes were calculated to be $1.07 \%$ and $0.43 \%$. Utilising this information and Equation (5), two simultaneous equations are obtained which can be solved to obtain $\alpha$ and $\beta$.

$$
\begin{aligned}
& 0.0107=\frac{\alpha}{2 \times(287.04 \times 2 \times \pi)}+\frac{\beta \times(287.04 \times 2 \times \pi)}{2} \\
& 0.0043=\frac{\alpha}{2 \times(1798.80 \times 2 \times \pi)}+\frac{\beta \times(1798.80 \times 2 \times \pi)}{2}
\end{aligned}
$$

From the solution to Equations 27 and 28, $\alpha$ and $\beta$ are calculated as $37.06 \mathrm{rad} / \mathrm{s}$ and $4.71 \times 10^{-7} \mathrm{~s} / \mathrm{rad}$, respectively. From Figure 11, it can be seen that as the beam length increases beyond a critical value the mechanical damping increases, suppressing the deflection and reducing the strain acting on the piezoelectric material. This mechanism is responsible for causing a reduction in the generated voltage and power for increasingly long beams. From Figure 10, a beam having length $3500 \mu \mathrm{m}$ generates $0.674 \mu \mathrm{W}$ for a $500 \mathrm{k} \Omega$ load resistance, whilst a beam having length $1850 \mu \mathrm{m}$ generates $0.876 \mu \mathrm{W}$, equating to a $30 \%$ increase. Maximum power, $3.717 \mu \mathrm{W}$, when excited at the natural frequency of the configuration, can be generated when a sample length of $1300 \mu \mathrm{m}$ is connected to a $34.1 \mathrm{k} \Omega$ load resistance.

Other researchers who have investigated the effect of beam length include Zhu et al [11] who used finite element software to develop a coupled piezoelectric-circuit finite element model. The main conclusion was that as the length of the beam is reduced, a 


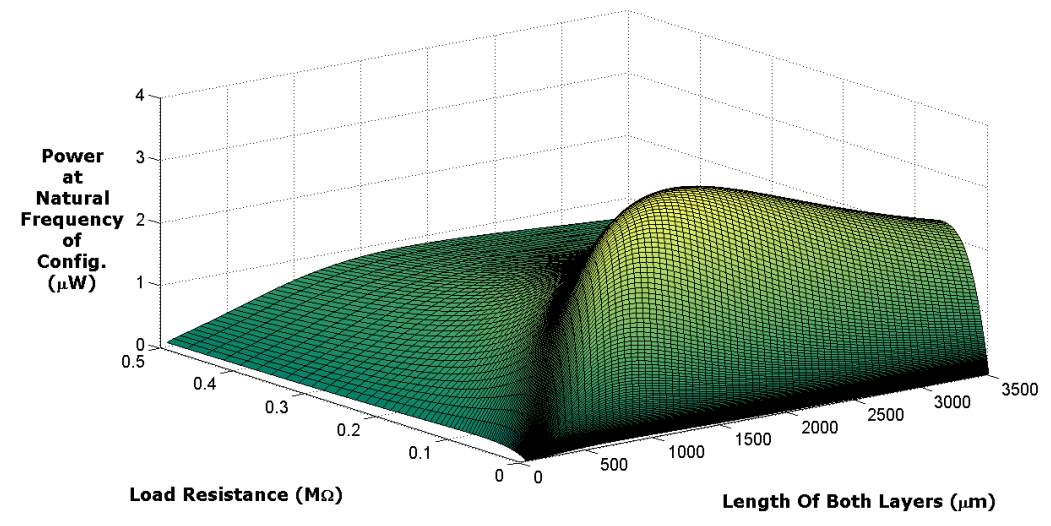

Figure 10: Plot showing how length of an energy harvester and load resistance magnitude affect the power dissipated. In this case both piezoelectric and substrate layers are the same length.

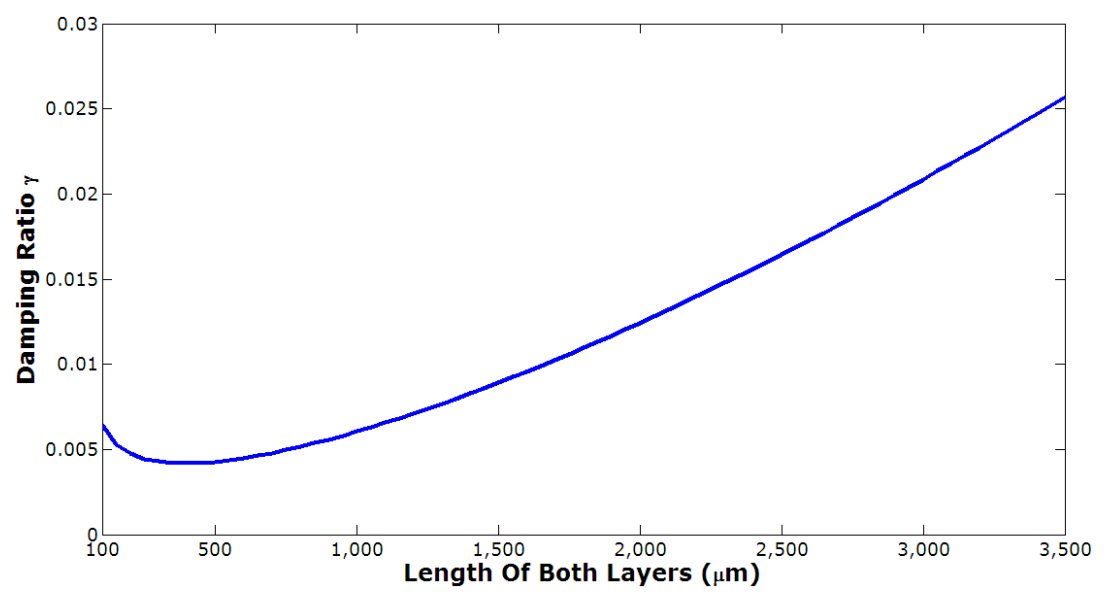

Figure 11: Plot showing how the length of an energy harvester affects the mechanical damping ratio, obtained using (Equation (5)). In this case both piezoelectric and substrate layers are of the same length.

continual increase in generated power is observed. The results presented here contradict this observation, and the reason for this was that Zhu et al incorrectly normalise their power results: their results are normalised by dividing with input acceleration instead of input acceleration squared. It is also worth noting that Zhu et al did not take account of how the mechanical damping ratio changes with beam length.

The second parameter under investigation is the length of the piezoelectric layer. Figure 12(a) shows the influence of using different lengths of PZT on the power. For a $500 \mathrm{k} \Omega$ load resistance a PZT layer that is only $520 \mu \mathrm{m}$ long dissipates $105 \%$ more power than a layer of length $2000 \mu \mathrm{m}$. The optimum load resistance for the conventional design was found to be $48 \mathrm{k} \Omega$, and using this resistance a power of $3.341 \mu \mathrm{W}$ is dissipated. For this particular device, maximum dissipated power was found to occur using PZT of length $1640 \mu \mathrm{m}$, which is only slightly larger than the maximum power generated by the 


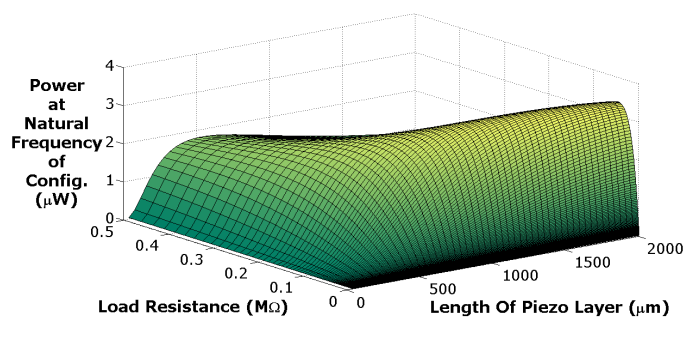

(a) Effect on dissipated power

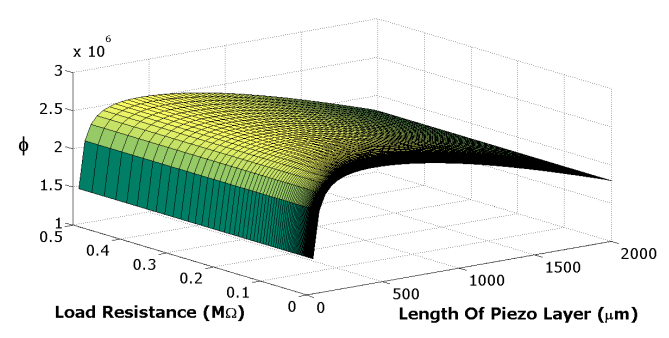

(b) Effect on electromechanical coupling term $\phi_{1}$

Figure 12: Plots showing how piezoelectric material length and load resistance magnitude affect certain variables.

conventional design. Recently, Bourisli and Al-Ajmi [12] investigated the influence of geometric parameters on the Electro-Mechanical Coupling Coefficient (EMCC), instead of the power. Their results indicated that a shorter piezoelectric layer is beneficial for increasing the EMCC. It had previously been shown by Renno et al [25] that a higher coupling coefficient will not necessarily benefit power generating capabilities of an energy harvester. The results from this model and research concur with this statement. As shown in Figure 12(b), $\phi_{1}$ is maximised when the PZT length is $440 \mu \mathrm{m}$, which does not correspond to maximum power. Due to the complex interactions between parameters it is difficult to single out and optimise one variable, i.e. $\phi_{1}$, while keeping all others at an optimum level. For this reason the authors opted to adjust physical parameters rather than individual equation variables.

Another parameter examined is the thickness of the piezoelectric layer, see Figure 13(a). The results indicate that increasing the thickness of the piezoelectric layer is advantageous for most load resistance magnitudes. If the PZT thickness is $6 \mu \mathrm{m}$ a power output of $4.535 \mu \mathrm{W}$ over a $60.3 \mathrm{k} \Omega$ resistor is achievable - equating to a $38.4 \%$ increase over a device with the nominal dimensions shown in Table 1 . The distance between the neutral axis and the piezoelectric material increases with an increase in PZT thickness. Greater strain is now exerted in the piezoelectric material thereby generating increased voltage. The piezoelectric internal capacitance, $C_{p}$, see Equation (11), reduces with increasing thickness which is also beneficial for voltage generation. These advantages are countered by an increase in structural stiffness causing the existence of an optimum thickness. Caution must be taken when increasing the thickness of the piezolayer as the resulting device may be more susceptible to fracture. Changing the thickness of the piezolayer changes the natural frequency, see Figure 13(b). A thickness increase from 1 to $4 \mu \mathrm{m}$ changes the fundamental frequency from $236.1 \mathrm{~Hz}$ to $282.4 \mathrm{~Hz}$. As expected, certain geometrical parameters cause greater changes in the natural frequency, such as beam length and layer thickness, whilst the influence of piezolayer length is predominantly dependent on the piezolayer thickness. The thicker the piezolayer the more its length will influence the natural frequency of the structure. 


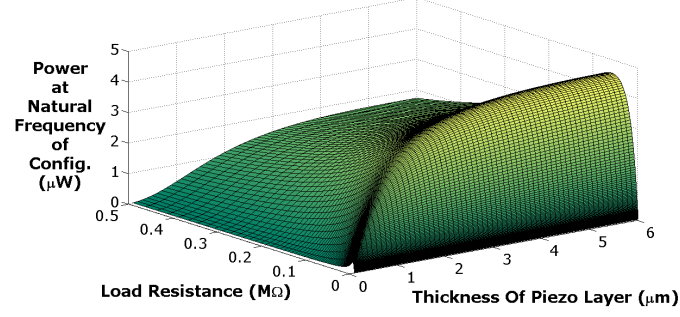

(a) Effect on dissipated power

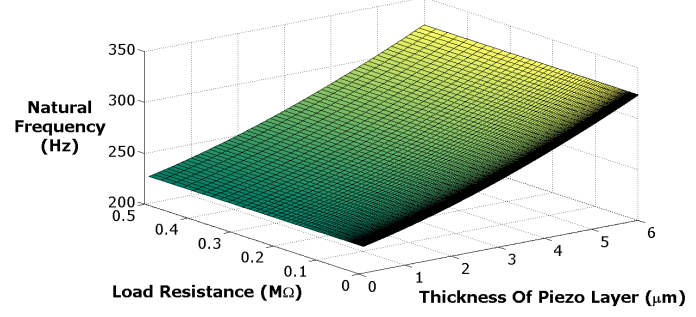

(b) Effect on natural frequency of the structure

Figure 13: Plots showing how piezoelectric material thickness and load resistance magnitude affect certain variables.

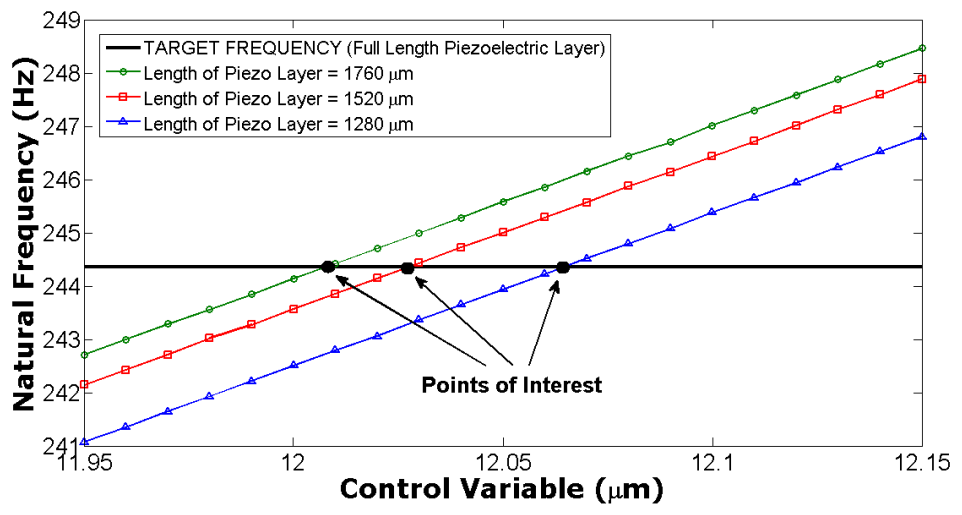

Figure 14: Plot showing an example of a good control parameter. In this case the substrate thickness.

\subsection{Constant-natural-frequency model}

Given that energy harvester performance is very much dependent on its natural frequency, it is rational to compare different energy harvester configurations having the same natural frequency. To achieve this some parameters are used as "control parameters" which are varied simultaneously with the other parameter under investigation. Example control parameters include: substrate thickness and width, the inertia of the tip mass, and material density. As an example, Figure 14 shows results obtained using substrate thickness as the control parameter. The parameter under investigation is the length of the piezoelectric layer from the clamped end. For each desired length, the control parameter (substrate thickness) is sampled and a search performed to determine the substrate thickness required to obtain the target natural frequency. Through this process a number of configurations can be generated with identical fundamental frequencies. The dimensions for some of these configurations can be found in Table 2 .

A similar approach is used by Gao et al [26] whereby the substrate length is used as the natural frequency control parameter. The group were investigating effects of 
Table 2: Structural dimensions of harvester configurations with identical natural frequencies. Obtained by sweeping substrate thickness for various lengths of PZT layer and using interpolation to determine desired thickness, Figure 14. Note: $\mathrm{L}_{\mathrm{m}}, \mathrm{b}_{\mathrm{m}}$ and $\mathrm{t}_{\mathrm{m}}$ refer to the tip mass length, width and thickness

\begin{tabular}{lllllllllll}
\hline $\begin{array}{l}\mathrm{L}_{\mathrm{b}} \\
(\mu \mathrm{m})\end{array}$ & $\begin{array}{l}\mathrm{x}_{1} \\
(\mu \mathrm{m})\end{array}$ & $\begin{array}{l}\mathrm{x}_{2} \\
(\mu \mathrm{m})\end{array}$ & $\begin{array}{l}\mathrm{x}_{3} \\
(\mu \mathrm{m})\end{array}$ & $\begin{array}{l}\mathrm{t}_{\mathrm{p}} \\
(\mu \mathrm{m})\end{array}$ & $\begin{array}{l}\mathbf{t}_{\mathrm{s}} \\
(\mu \mathrm{m})\end{array}$ & $\begin{array}{l}\mathrm{b}_{\mathrm{p}} \\
(\mu \mathrm{m})\end{array}$ & $\begin{array}{l}\mathrm{b}_{\mathrm{s}} \\
(\mu \mathrm{m})\end{array}$ & $\begin{array}{l}\mathrm{L}_{\mathrm{m}} \\
(\mu \mathrm{m})\end{array}$ & $\begin{array}{l}\mathrm{b}_{\mathrm{m}} \\
(\mu \mathrm{m})\end{array}$ & $\begin{array}{l}\mathrm{t}_{\mathrm{m}} \\
(\mu \mathrm{m})\end{array}$ \\
\hline 2000 & 0 & $\mathbf{2 0 0 0}$ & $\mathbf{0}$ & 1.64 & $\mathbf{1 2 . 0 0 0}$ & 600 & 600 & 600 & 600 & 600 \\
2000 & 0 & $\mathbf{1 6 0 0}$ & $\mathbf{4 0 0}$ & 1.64 & $\mathbf{1 2 . 0 1 9}$ & 600 & 600 & 600 & 600 & 600 \\
2000 & 0 & $\mathbf{1 2 0 0}$ & $\mathbf{8 0 0}$ & 1.64 & $\mathbf{1 2 . 0 8 2}$ & 600 & 600 & 600 & 600 & 600 \\
2000 & 0 & $\mathbf{8 0 0}$ & $\mathbf{1 2 0 0}$ & 1.64 & $\mathbf{1 2 . 2 0 5}$ & 600 & 600 & 600 & 600 & 600 \\
\hline
\end{tabular}

the length ratio between the substrate and piezoelectric layers on voltage and power generation. Findings showed a length ratio of 1 (both layer are equal in length) is ideal for maximising power, whereas a ratio of more than 1 (longer substrate layer) allows for optimised peak voltage.

\subsection{Changing multiple parameters}

In this section, multiple parameters are adjusted simultaneously in order to maintain a constant target natural frequency, $244.4 \mathrm{~Hz} .244 .4 \mathrm{~Hz}$ is chosen due to the fact that this is the natural frequency of the conventional configuration. It is assumed the conventional configuration would be designed for a specific application with known excitation frequency. Therefore it is only logical to 'tune' the natural frequency of other configurations to this. Figure 15 shows how the length of the piezoelectric layer affects the power, while the substrate layer is used as a frequency control variable. For each increment in configuration number the piezoelectric layer is shortened by $40 \mu \mathrm{m}$. Figure 16 shows the piezoelectric layer length and substrate thickness for all examined configurations. From configuration C1 (conventional design) to configuration C20 (PZT length $1000 \mu \mathrm{m}$ ), the power output is seen to remain relatively constant - difference of only $2.74 \%$. The optimum configuration is configuration $\mathrm{C} 10$ where $3.415 \mu \mathrm{W}$ is dissipated through a $57.5 \mathrm{k} \Omega$ resistor. For a load resistance of $500 \mathrm{k} \Omega$, configuration C38 can dissipate $102 \%$ more power in comparison to the conventional design. The dimensions for configurations $\mathrm{C} 10$ and $\mathrm{C} 38$ are shown in Table 3.

Results were also performed using tip mass inertia as the control variable, see Figure 17. The results obtained while changing the length of the piezoelectric layer show similar trends to those when the substrate thickness is used as the control variable. This is because there is little difference between how the two control parameters affect the mechanical behaviour of the system. Figure 18 can be referred to for the piezoelectric material length and the tip mass length and thickness for each configuration number.

By looking at the effect of piezoelectric layer thickness, Figure 17(b), one can 


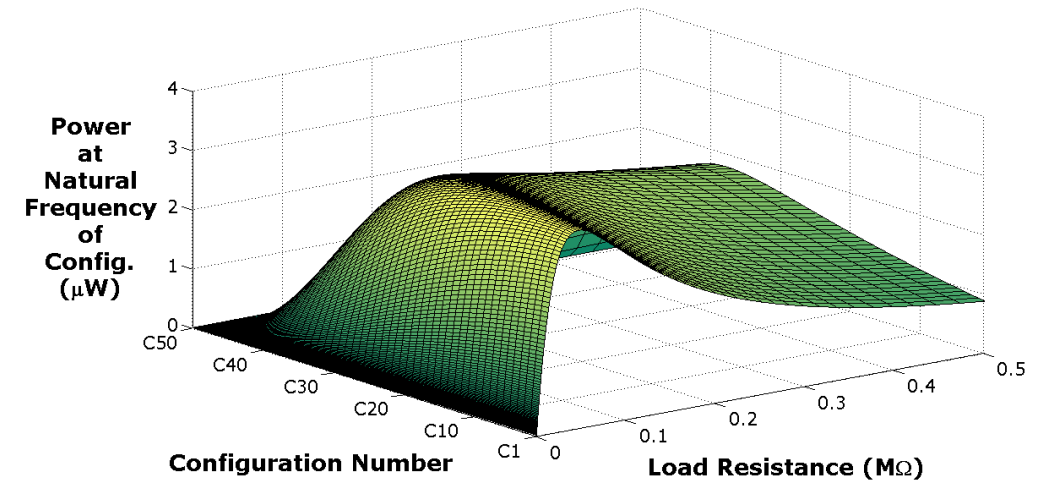

Figure 15: Plot showing how piezoelectric material length and load resistance magnitude affect the power generated while substrate thickness is used for frequency control.

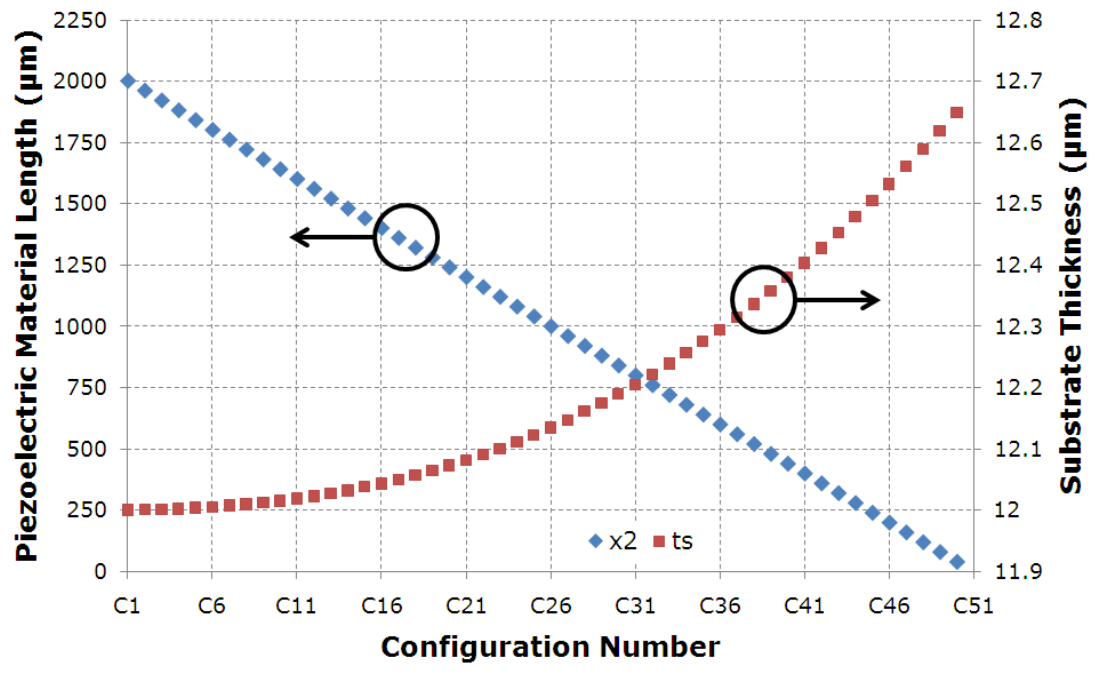

Figure 16: Plot showing piezoelectric length and substrate thickness for each configuration number corresponding to Figure 15.

Table 3: Structural dimensions of harvester configurations with identical natural frequencies. Substrate thickness is used as the control variable.

\begin{tabular}{llllllllllll}
\hline Config. & $\begin{array}{l}\mathrm{L}_{\mathrm{b}} \\
(\mu \mathrm{m})\end{array}$ & $\begin{array}{l}\mathrm{x}_{1} \\
(\mu \mathrm{m})\end{array}$ & $\begin{array}{l}\mathrm{x}_{2} \\
(\mu \mathrm{m})\end{array}$ & $\begin{array}{l}\mathrm{x}_{3} \\
(\mu \mathrm{m})\end{array}$ & $\begin{array}{l}\mathrm{t}_{\mathrm{p}} \\
(\mu \mathrm{m})\end{array}$ & $\begin{array}{l}\mathbf{t}_{\mathrm{s}} \\
(\mu \mathrm{m})\end{array}$ & $\begin{array}{l}\mathrm{b}_{\mathrm{p}} \\
(\mu \mathrm{m})\end{array}$ & $\begin{array}{l}\mathrm{b}_{\mathrm{s}} \\
(\mu \mathrm{m})\end{array}$ & $\begin{array}{l}\mathrm{L}_{\mathrm{m}} \\
(\mu \mathrm{m})\end{array}$ & $\begin{array}{l}\mathrm{b}_{\mathrm{m}} \\
(\mu \mathrm{m})\end{array}$ & $\begin{array}{l}\mathrm{t}_{\mathrm{m}} \\
(\mu \mathrm{m})\end{array}$ \\
\hline $\mathrm{C} 10$ & 2000 & 0 & $\mathbf{1 6 4 0}$ & $\mathbf{3 6 0}$ & 1.64 & $\mathbf{1 2 . 0 1 5}$ & 600 & 600 & 600 & 600 & 600 \\
$\mathrm{C} 38$ & 2000 & 0 & $\mathbf{5 2 0}$ & $\mathbf{1 4 8 0}$ & 1.64 & $\mathbf{1 2 . 3 3 6}$ & 600 & 600 & 600 & 600 & 600 \\
$\mathrm{C} 44$ & 2000 & 0 & $\mathbf{2 8 0}$ & $\mathbf{1 7 2 0}$ & 1.64 & $\mathbf{1 2 . 4 7 9}$ & 600 & 600 & 600 & 600 & 600 \\
\hline
\end{tabular}




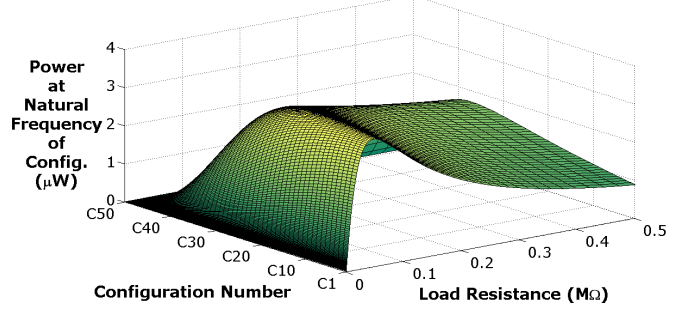

(a) Effect of PZT length

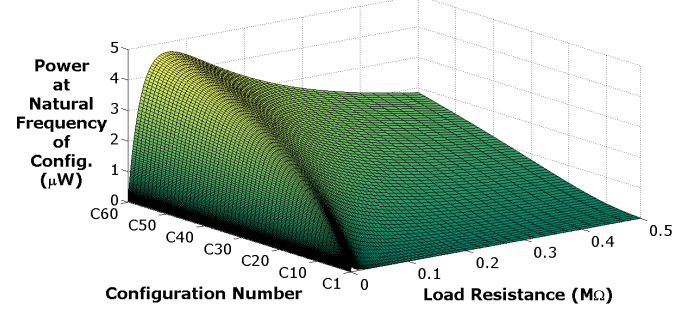

(b) Effect of PZT thickness

Figure 17: Plots showing the trends in power dissipated while using tip mass inertia as the control parameter.

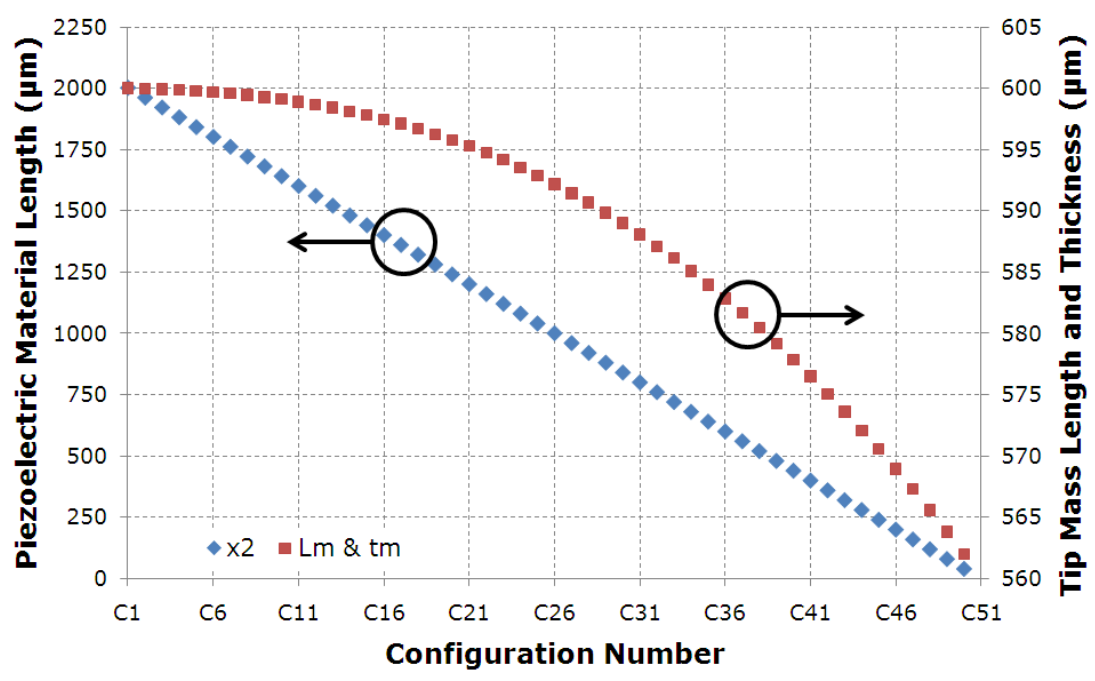

Figure 18: Plot showing piezoelectric length and tip mass length and thickness for each configuration number corresponding to Figure 17(a).

deduce that a thicker PZT layer is beneficial for energy harvesting. While increasing thickness, the stiffness of the beam increases together with the natural frequency; this is countered by increasing the inertia of the tip mass. For the conventional configuration the piezoelectric layer thickness is $1.64 \mu \mathrm{m}$ and the tip mass measures $600 \mu \mathrm{m} \times 600 \mu \mathrm{m}$ $\times 600 \mu \mathrm{m}$. In comparison, configuration $\mathrm{C} 60$ has a $3 \mu \mathrm{m}$ thick piezoelectric layer and is comprises of a $641.23 \mu \mathrm{m} \times 600 \mu \mathrm{m} \times 641.23 \mu \mathrm{m}$ tip mass. The difference in maximum dissipated power between these two configurations is $39.4 \%$. The piezoelectric layer thickness and tip mass length and thickness for all other configurations can be found in Figure 19.

\subsection{Charging circuit}

The constant frequency model described in Section 3.2 is combined with the capacitor charging model described in Section 2.3. Note, when simulating the harvester connected to the charging circuit the input acceleration is increased to $3 \mathrm{~g}$. This allows for sufficient 


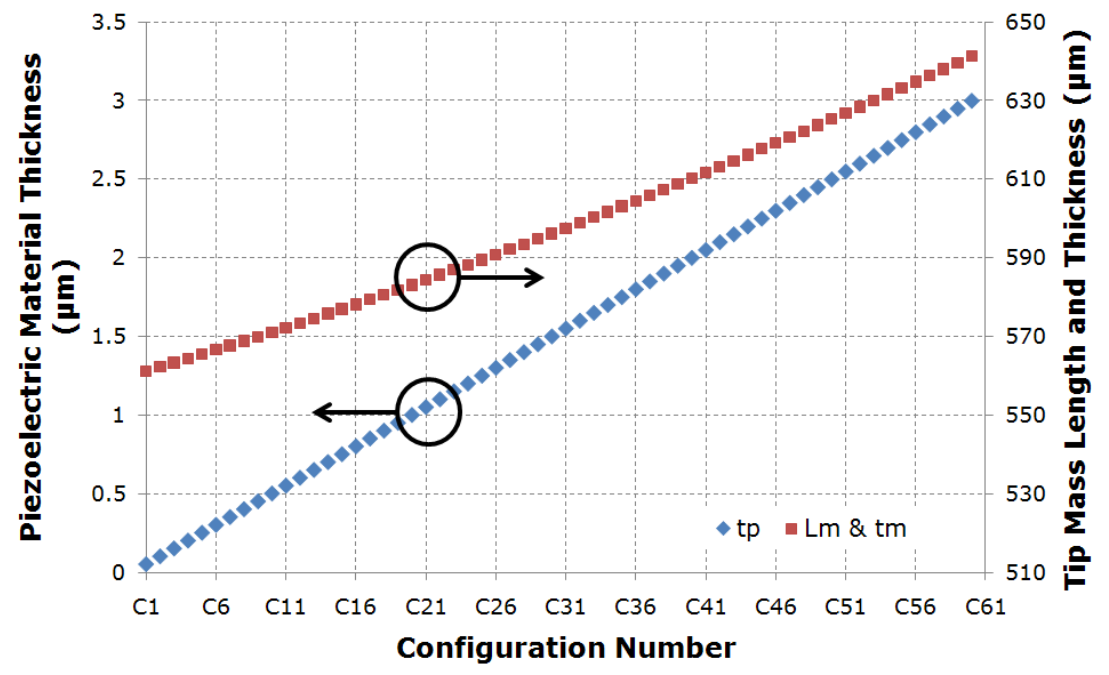

Figure 19: Plot showing piezoelectric thickness and tip mass length and thickness for each configuration number corresponding to Figure 17(b).

voltage generation to overcome the diode threshold voltage, $\mathrm{V}_{\mathrm{th}}$. Figure 20 shows results when the length of the piezoelectric layer is altered, while using the substrate thickness as the control variable. The results illustrate that a shorter piezoelectric layer is beneficial in terms of voltage for all time periods. After $45 \mathrm{~s}$, an increase of $98 \%$ in the voltage across a $1 \mathrm{nF}$ capacitor is possible if configuration C44 is used instead of the conventional design. Configuration C44 consists of a $280 \mu \mathrm{m}$ PZT layer attached to a $12.48 \mu \mathrm{m}$ thick silicon layer and allows for $1.410 \mathrm{~V}$ to be stored after $45 \mathrm{~s}$. By looking at the equations governing the system, the reasons for this behaviour becomes apparent. When the PZT layer is shortened, the piezoelectric internal capacitance reduces, refer to Equation (11). This is beneficial because the current flowing through and the rate of change of voltage across the storage capacitor increase, see Equation (26). The disadvantage of reducing the PZT length is that the generated current decreases, see Figure 21. It is the magnitude by which each of these changes occurs which determines whether the stored voltage will increase or decrease.

Figure 22 shows the relationship between piezoelectric layer thickness and voltage, when the tip mass is used as the control parameter. The results indicate a similar trend to that obtained using the load resistance model. After $45 \mathrm{~s}$, configuration C20 will charge a $1 \mathrm{nF}$ capacitor to $0.364 \mathrm{~V}$, whereas configuration C60 charges the capacitor to $1.24 \mathrm{~V}$, a $241 \%$ increase. Configuration C20 comprises a $1 \mu \mathrm{m}$ thick piezoelectric layer and a $583.11 \mu \mathrm{m} \times 600 \mu \mathrm{m} \times 583.11 \mu \mathrm{m}$ tip mass.

\section{Conclusion}

A comprehensive model for a piezoelectric cantilever beam energy harvester has been developed which is well suited to performing a wide range of parameter studies. It is realised by combining a finite element model of the mechanical structure and a 


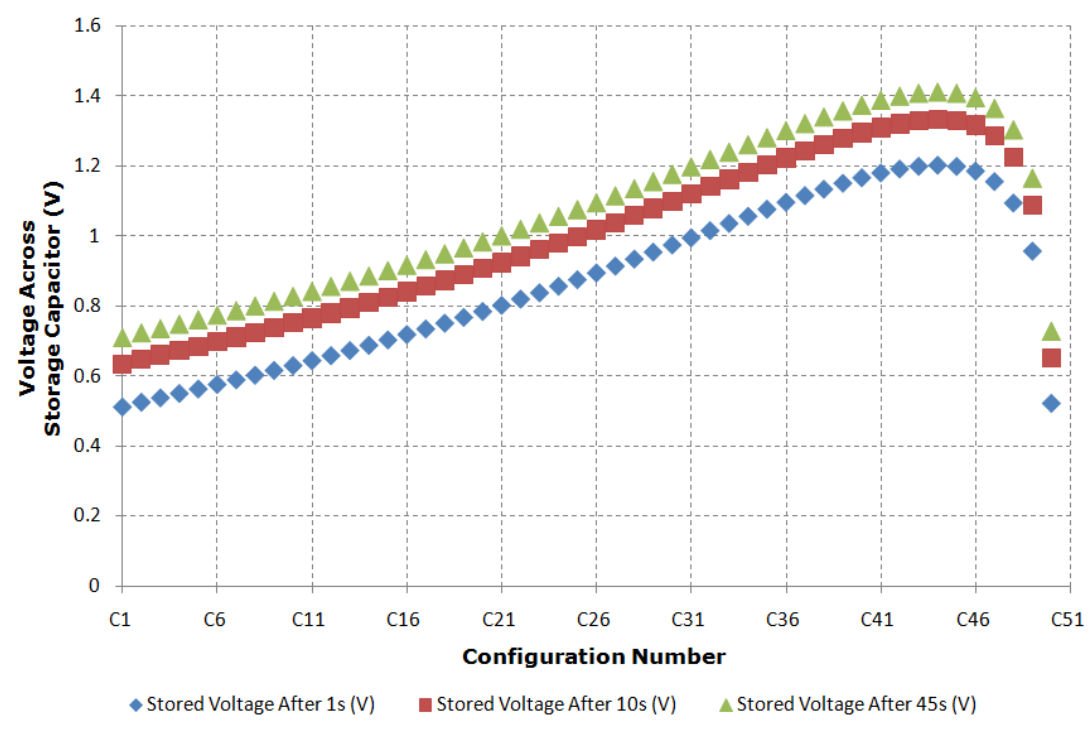

Figure 20: Plot showing how piezoelectric material length affects the voltage across a $1 \mathrm{nF}$ capacitor. The substrate thickness is used for frequency control.

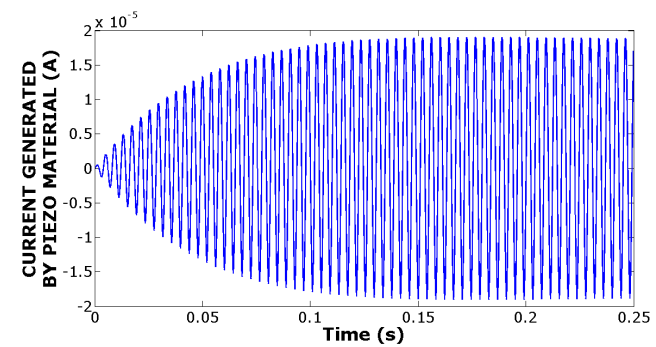

(a) Configuration $\mathrm{C} 1$

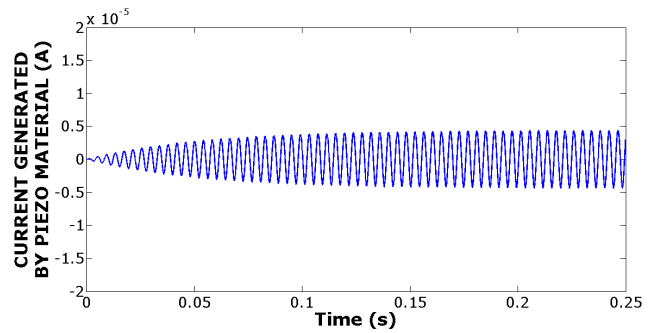

(b) Configuration C44, Table 3

Figure 21: Current generated by the piezoelectric material during the first $0.25 \mathrm{~s}$ for two configurations with identical fundamental frequencies. Substrate thickness used as control variable.

distributed parameter electromechanical model. The mechanical model allows the position and length of the piezoelectric layer to be varied, and takes into account changes in the mechanical damping ratio as the dimensions of the harvester are changed. The standard load resistor model for the electrical circuit has been extended to allow energy to be stored across a capacitor. In addition, the model allows individual and multiple parameters to be varied and compared simultaneously.

For the standard resistor load model, the obtained results indicate that an optimum beam length exists due to increased mechanical damping in longer beams, and the piezoelectric thickness is observed to have a significant influence on dissipated power. In addition, the magnitude of the load resistance is found to play an important role. The observed trends in dissipated power, as parameters are altered, are heavily dependant on the load resistance due to intricate coupling between the electrical and mechanical aspect of the system. 


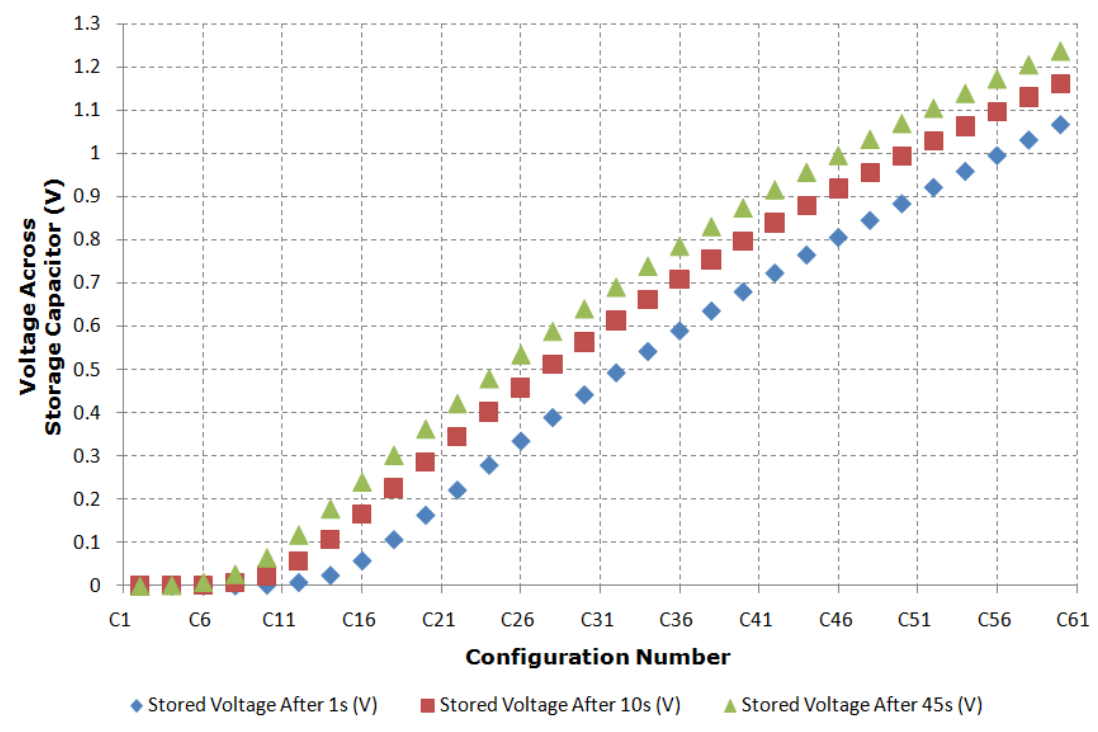

Figure 22: Plot showing how piezoelectric material thickness affects the voltage across a $1 \mathrm{nF}$ capacitor. The tip mass inertia is used for frequency control.

When performing geometric parameter studies it is clear that the power generated is very much dependent on the natural frequency of the dominant mode, and the natural frequency changes as the geometry is changed. To overcome this issue and facilitate a more rational approach to comparing the performance of different harvester designs, a control parameter concept was introduced that allowed different harvester configurations to be generated, with each configuration having the same natural frequency. It was found that substrate thickness and tip mass inertia could be used as effective control parameters.

The most interesting results were observed when examining the effects of PZT geometry on energy storage. Significant increases compared to the conventional design were observed when reducing piezoelectric layer length. These gains are due to a reduction in the internal capacitance of the piezoelectric layer. Similar performance enhancements were achieved by varying the thickness of the PZT layer and using tip mass inertia to maintain the natural frequency.

Although the work presented here indicates that performance enhancements are possible by modifying the piezoelectric layer geometry, it is important to realise that optimum dimensions achieved for the piezoelectric material will not always guarantee improvements. The intricate coupling between the mechanical and electrical domains makes it virtually impossible to determine a universal configuration that maximises performance for all application areas. Once basic requirements are known, for example the space envelope, materials and excitation signal properties, an optimisation process should be undertaken. 


\section{Acknowledgments}

This work was supported by the Engineering and Physical Sciences Research Council

of the U.K. A special thank you is accredited Dr Adam Clare for his support regarding the experimental work.

\section{References}

[1] Roundy S 2008 Proc. of PowerMEMS 2008 (Sendai, Japan) p 1-4

[2] Kulkarni S, Koukharenko E, Torah R, Tudor J, Beeby S, O'Donnel T and Roy S 2008 Sensor. Actuator. 145-146 336-342

[3] Chiu Y, Kuo C T and Chu Y S 2007 Microsyst. Technol. 13 1663-1669

[4] Elfrink R, Kamel T M, Goedbloed M, Matova S, Hohlfeld D, van Andel Y and van Schaijk R 2009 J. Micromech. Microeng. 19094005

[5] Roundy S, Leland E S, Baker J, Carleton E, Reilly E, Lai E, Otis B, Rabaey J M, Wright P K and Sundararajan V 2005 IEEE Pervas. Comput. 4 28-36

[6] Priya S and Inman D 2009 Energy Harvesting Technologies (New York: Springer Science+Business Media, LLC)

[7] Lin J H, Wu X M, Ren T L and Liu L T 2007 Integr. Ferroelectr. 95 128-141

[8] Gao X, Shih W H and Shih W Y 2009 Smart Mater. Struct. 18125018

[9] Friswell M I and Adhikari S 2010 J. Appl. Phys. 108014901

[10] Chen Z S, Yang Y M and Deng G Q 2009 Sustainable Power Generation and Supply 2009 (Nanjing, China) p 1-6

[11] Zhu M, Worthington E and Tiwari A 2010 IEEE T. Ultrason. Ferr. 57 427-437

[12] Bourisli R I and Al-Ajmi M A 2010 J. Intel. Mat. Syst. Str. 21 907-914

[13] Sodano H A, Inman D J and Park G 2005 J. Intel. Mat. Syst. Str. 16 799-807

[14] Williams C B and Yates R B 1996 Sensor. Actuator. 52 8-11

[15] Sodano H A, Park G and Inman D J 2004 Strain 40 49-58

[16] Erturk A and Inman D J 2008 Smart Mater. Struct. 17065016

[17] Erturk A and Inman D J 2008 J. Vib. Acoust. 130041002

[18] Erturk A and Inman D J 2009 Smart Mater. Struct. 18025009

[19] Rao S S 2004 Mechanical Vibrations, Fourth Edition (New Jersey: Prentice Education, Inc.) p 862$865 \&$ p 234

[20] MathWorks http://www.mathworks.com/help/toolbox/physmod/simscape/ $5^{\text {th }}$ August 2010

[21] MathWorks http://www.mathworks.com/help/toolbox/physmod/elec/ $5^{\text {th }}$ August 2010

[22] Fang H B, Liu J Q, Xu Z Y, Dong L,Wang L, Chen D, Cai B C and Liu Y 2006 Microelectr. J. 37 1280-1284

[23] ABAQUS CAE http://caunton/v6.7/index.html $5^{\text {th }}$ March 2009

[24] Patel R, McWilliam S and Popov A A 2010 Proc. of PowerMEMS 2010 (Leuven, Belgium) p 395398

[25] Renno J M, Daqaq M F and Inman D J 2009 J. Sound Vib. 320 386-405

[26] Gao X, Shih W H and Shih W Y 2010 Appl. Phys. Lett. 97233503 